\title{
ORIGINAL
}

\section{EFICACIA DE UNA INTERVENCIÓN EDUCATIVA GRUPAL SOBRE CAMBIOS EN LOS ESTILOS DE VIDA EN HIPERTENSOS EN ATENCIÓN PRIMARIA: UN ENSAYO CLÍNICO ALEATORIO (*)}

\author{
Carmela Rodríguez Martín, Carmen Castaño Sánchez, Luis García Ortiz, José Ignacio Recio \\ Rodríguez, Yolanda Castaño Sánchez y Manuel Ángel Gómez Marcos
}

Unidad de Investigación. Centro de salud La Alamedilla. Salamanca.

\section{RESUMEN}

Fundamentos: Los estilos de vida saludables se relacionan con un menor riesgo de enfermedades cardiovasculares. El objetivo del estudio es evaluar la eficacia de una estrategia educativa grupal en la modificación de estilos de vida, control de factores de riesgo y riesgo cardiovascular (RCV) en personas hipertensas.

Método: Ensayo clínico aleatorio en Atención Primaria. Se seleccionaron por muestreo aleatorio 101 personas hipertensas de 35 a 74 años, randomizando 51 al grupo de intervención (GI)(64,5 $\pm 9,7$ años, 56\% mujeres) y 50 al control (GC) (65,4 $\pm 8,4$ años, $68 \%$ mujeres). Se realizó una evaluación basal, una intervención educativa grupal sobre cambios de estilos de vida (seis sesiones durante un año) y una evaluación final. El efecto de la intervención se evaluó con RCV (Framingham), presión arterial(PA), perfil lipídico, perímetro de cintura, índice de masa corporal(IMC), consumo de nutrientes con encuesta validada, ejercicio físico con 7-PAR Day y calidad de vida con SF-36.

Resultados: PA basal 136,8/82,7 mmHg en GI y 139,3/79,3 mmHg y en GC, RCV:11,1\% (GI) y $12,3 \%$ (GC). La PA sistólica descendió $5,6 \pm 19,6 \mathrm{mmHg}(\mathrm{p}=0,07)$ en GI, y $7,1 \pm 16, \mathrm{mmHg}$ $(\mathrm{p}=0,004)$ en $\mathrm{GC}$, la diastólica $3,9 \pm 10,8(\mathrm{p}=0,02)$, y $2,7 \pm 11$, mmHg $(\mathrm{p}=0,10)$ respectivamente. El IMC descendió $0,3 \pm 1,6$ puntos en $\mathrm{GI}(\mathrm{p}=0,17)$, y aumentó $0,1 \pm 1,5$ en $\mathrm{GC}(\mathrm{p}=0,81)$. El RCV descendió $0,8 \pm 6,5$ puntos en GI y aumentó $0,2 \pm 6,8$ en GC, el efecto de la intervención fue un descenso de 1 punto (IC95\%:$3,9-1,9)(\mathrm{p}=0,48)$. La ingesta disminuyó en GI: $42,8 \pm 1141,2$ $\mathrm{Kcal} / \mathrm{día}(\mathrm{p}=0,14)$, y en GC: $278,9 \pm 1115,9(\mathrm{p}=0,62)$. El ejercicio (mets/hora/semana) aumentó en ambos, 3,6 19 en $\mathrm{GI}(\mathrm{p}=0,20)$ y $3,9 \pm 14,9$ en $\mathrm{GC}(\mathrm{p}=0,07)$.

Conclusión: Encontramos mayor descenso del riesgo cardiovascular en el grupo de intervención, sin existir diferencias estadísticamente significativas en los parámetros evaluados entre ambos grupos.

Palabras clave: Educación para la salud. Hipertensión arterial. Atención primaria de salud.

\section{Correspondencia:}

Carmela Rodríguez Martín.

Centro de salud La Alamedilla. Unidad de Investigación

Av. Comuneros 27-31

37003 Salamanca. España.

Correo electrónico: Lgarciao@usal.es

\section{ABSTRACT}

\section{Effycacy of an Educational Intervention Group on Changes in Lifestyles in Hypertensive Patients in Primary Care: A Randomized Clinical Trial}

Background: Healthy lifestyles are associated with less risk of cardiovascular disease. The aim of this study is to evaluate the effectiveness of a group educational strategy in lifestyle changes, as well as the control of risk factors and cardiovascular risk in hypertensive patients.

Methods: Randomized clinical trial carried out in Primary Care. 101 hypertensive patients were selected by random sampling, aged $35-74 ; 51$ patients were randomized to the intervention group (IG) (aged: 64,5 $\pm 9,7,56 \%$ women) and other 50 to the control group(CG)(aged: $65,4 \pm 8,4,68 \%$ women). We performed a basal evaluation and an educational intervention on lifestyles, six sessions during one year, and final-point evaluation. Effect of intervention was evaluated through of cardiovascular risk (Framingham), blood pressure, lipid profile, waist circumference, body mass index (BMI), nutrient consumption, physical exercise (7-PAR day) and quality of life(SF-36).

Results: Basal blood pressure was 136,8/82,7 mmHg IG and $139,3 / 79,3$ CG, cardiovascular risk was $11,1 \%$ y $12,3 \%$ respectively. Systolic blood pressure decreased 5,6 $\pm 19,6(\mathrm{p}=0,07)$ IG and 7,1 $\pm 16,3 \mathrm{mmHg}(\mathrm{p}=0,004) \mathrm{GC}$, and diastolic decreased $3,9 \pm 10,8(\mathrm{p}=0,02)$ and $2,7 \pm 11,5 \mathrm{mmHg}(\mathrm{p}=0,10)$ respectively. BMI decreased $0,3 \pm 1,6$ points IG $(\mathrm{p}=0,17)$ and increased $0,1 \pm 1,5$ $\mathrm{CG}(\mathrm{p}=0,81)$. Coronary risk decreased $0,8 \pm 6,5$ points IG and increased $0,2 \pm 6,8 \mathrm{CG}$; effect of intervention was a reduction in 1 point $(\mathrm{CI} 95 \%-3,9 \div 1,9)(\mathrm{p}=0,48)$. Calories ingestion decreased $42,8 \pm 1141,2 \mathrm{Kcal} / \mathrm{day} \mathrm{p}=0,14) \mathrm{IG}$ and $278,9 \pm 1115,9(\mathrm{p}=0,62)$ CG. Physical exercise increased in both groups: $3,6 \pm 19$ IG $(\mathrm{p}=0,20)$ and $3,9 \pm 14,9$ mets/hour/week CG $(\mathrm{p}=0,07)$.

Conclusions: There was a higher decline of cardiovascular risk in the intervention group than control group, we did not find statistically significant differences between both groups in parameters evaluated.

Key words: Health Education. Hypertension. Primary Health Care.

(*) Este proyecto ha sido financiado por la Consejería de Sanidad de la Junta de Castilla y León y el Instituto de Salud Carlos III (ISCIII-RETICS RD 06/0018). 


\section{INTRODUCCIÓN}

La hipertensión arterial (HTA) es el factor de riesgo cardiovascular (FRCV) con mayor prevalencia en nuestro medio, relacionándose con 1 de cada 4 muertes totales y 1 de cada 2,5 muertes de origen cardiovascular en España ${ }^{1}$. El control de la presión arterial ha mejorado en España durante los últimos años, no obstante todavía existe un amplio margen para mejorar ${ }^{2,3}$. La adopción de estilos de vida cardiosaludables se ha mostrado como una herramienta fundamental para prevenir la elevación de la presión arterial (PA) en población general y para mejorar el control de la misma en las personas hipertensas ${ }^{4}$.

Determinados cambios en los estilos de vida se han mostrado eficaces en el descenso de la presión arterial y deberían formar parte del abordaje terapéutico de las personas hipertensas. La pérdida de peso en las personas obesas ${ }^{5}$, el aumento del ejercicio físico aeróbico (caminar rápido al menos 30 minutos al día la mayoría de los días de la semana $)^{6}$ y la disminución del consumo de alcohol en las personas que beben cantidades excesivas ${ }^{7}$ se han mostrado eficaces en la reducción de la presión arterial. Asimismo, la reducción del consumo de sodio y una dieta baja en grasa total, ácidos grasos saturados y colesterol y rica en frutas, vegetales y productos lácteos desnatados ha mostrado reducciones de la $\mathrm{PA}^{8,9}$. En diferentes ensayos clínicos controlados se ha demostrado que estos cambios en los estilos de vida, mantenidos en el tiempo (más de 3 años), pueden disminuir las cifras de PA en pacientes hipertensos ${ }^{10}$.

Desde hace 8 años en el centro de salud del estudio se vienen realizando ciclos de mejora de calidad sobre los profesionales de atención primaria para mejorar el control de la hipertensión. Estos ciclos de calidad han mostrando eficacia en la mejora, tanto de los indicadores de proceso como de resultados intermedios ${ }^{11-15}$.
Las estrategias educativas realizadas por profesionales de la salud para mejorar el control de la presión arterial han ofrecido resultados heterogéneos ${ }^{16}$. Por otro lado la eficacia de una intervención educativa grupal sobre los estilos de vida de las personas hipertensas para mejorar el control y reducir el riesgo cardiovascular (RCV) global a medio y largo plazo no ha sido estudiada en nuestro medio.

El objetivo de este estudio es evaluar la eficacia a medio plazo de una estrategia educativa grupal en la modificación de los estilos de vida, el control de los factores de riesgo y el riesgo cardiovascular en personas con hipertensión.

\section{SUJETOS Y MÉTODO}

Diseño y ámbito de estudio: Estudio experimental, aleatorizado y controlado, desarrollado en un centro de salud urbano de Castilla y León.

Sujetos del estudio: El tamaño de la muestra se estimó para detectar una diferencia mayor o igual a $8 \mathrm{~mm} \mathrm{Hg}$ en la presión arterial sistólica (PAS), que es el descenso conseguido con la dieta DASH (equivalente a la dieta mediterránea) ${ }^{10}$. Aceptando un riesgo alfa de 0,05 y un riesgo beta de 0,20 para un contraste bilateral con una desviación estándar de 14 $\mathrm{mmHg}^{13}$ y estimando que las pérdidas del seguimiento serán de un 5\% se precisan 51 sujetos en cada grupo.

De los 2.180 sujetos hipertensos de entre 35 y 74 años registrados, se seleccionaron por muestreo aleatorio simple y se asignaron mediante el programa Epidat versión 3.1. un total de 110. Fueron eliminados de la muestra aquéllos que no cumplían los criterios de inclusión establecidos y al resto se les ofreció participar en el estudio. Los sujetos que firmaron el consentimiento informado fueron aleatoriza- 
dos en los grupos de intervención (GI) y de control (GC), mediante el mismo programa informático.

Se excluyeron los pacientes con cardiopatía isquémica, insuficiencia cardiaca, miocardiopatía, accidente cerebro vascular con secuelas, EPOC severa o moderada, enfermedad mental, demencia, alcoholismo, cáncer en tratamiento en los últimos 5 años, inmovilizados o terminales, enfermedad reumática severa, otra patología severa que le impidiese participar y haber sido diagnosticado de HTA en un periodo inferior a 6 meses.

Fases del estudio: 1. Formación de los profesionales. Durante el primer trimestre de 2005 mediante sesiones del equipo investigador (3 médicos y 4 enfermeras) se analizó la literatura científica y se elaboró una intervención estructurada y uniforme. Para ello se revisaron las guías de práctica clínica sobre hipertensión arterial y otros factores de riesgo cardiovascular ${ }^{10,17-21}$ utilizadas habitualmente en nuestro entorno. Se realizó un adiestramiento en el uso de las escalas de estimación de $\mathrm{RCV}^{22}$, en las escalas de calidad de vida ${ }^{23}$ y en las herramientas para evaluar la actividad física ${ }^{24}$ y el patrón alimentario ${ }^{25}$. También se revisó el modelo transteórico de Prochaska y Diclemente ${ }^{26}$ y se realizó un entrenamiento en técnicas de educación grupal (grupos de discusión dirigida). Se realizó una prueba piloto para evaluar la correcta capacitación y la homogeneización de los criterios en la intervención, así como para valorar la estrategia final.

2. Evaluación inicial: realizada durante el $2^{\circ}$ semestre del 2005. Se cumplimentaron los cuestionarios sobre calidad de vida, alimentación, motivación, y se realizaron las exploraciones necesarias para valorar la actividad física, los estilos de vida y la situación de los factores de riesgo y el riesgo cardiovascular de los dos grupos.
3. Fase de Intervención: Las actividades educativas sobre los estilos de vida se realizaron en los pacientes hipertensos del grupo de intervención a lo largo del año 2006. Estos se distribuyeron en 5 grupos homogéneos de 10-12 pacientes. En cada grupo se realizaron 3 sesiones de discusión dirigida de 1 hora de duración, 2 talleres prácticos de 2 horas y una sesión de refuerzo un mes después para trabajar las dificultades en el cumplimiento. En todas ellas se fomentó la participación, el compartir experiencias y la retroalimentación de los participantes.

En la $1^{a}$ sesión (enero y febrero de 2006), se trabajó el concepto de riesgo y factores de riesgo cardiovascular y las medidas higiénico-dietéticas para disminuir la presión arterial y el riesgo, partiendo de experiencias concretas conocidas por los miembros del grupo.

En la $2^{\text {a }}$ sesión (marzo y abril de 2006), partiendo de los alimentos que habían comido el día anterior, se reflexionó sobre el concepto de dieta sana y los beneficios de la dieta mediterránea en la salud cardiovascular $^{27}$. Se reforzó el concepto de dieta sosa y la importancia del consumo moderado de alcohol $^{7,9}$. Posteriormente, se recogieron las dificultades para seguir una alimentación sana.

En la $3^{\text {a }}$ sesión (mayo de 2006), se profundizó en el tabaco como principal causa evitable de muerte prematura. También se abordó la importancia del ejercicio físico, beneficios y dificultades que se tienen para realizarlo, y diferentes técnicas de relajación para controlar el estrés.

Taller de alimentación (junio del 2006), con la participación de profesionales expertos en nutrición, se entrenó a los pacientes en la confección de una dieta cardiosaludable ${ }^{28}$ y se realizó una actividad práctica cocinando y degustando diferentes alimentos. 
Taller de actividad física (septiembre de 2006), dos expertos en ejercicio revisaron las fases de la actividad física y el tipo de ejercicio recomendado para cada integrante del grupo de estudio dependiendo de su condición física ${ }^{6}$.

Durante el mes de diciembre de 2006 se llevó a cabo una sesión de refuerzo trabajando con los pacientes las dificultades en el cumplimiento de la dieta y el ejercicio, reforzando su importancia y ofertando soluciones para mejorarlo.

Durante el año 2007 no se realizó ninguna intervención para poder valorar un año después la eficacia de la intervención, así como la permanecía en el tiempo del efecto.

4. Evaluación final: Durante el primer trimestre del 2008 se realizó una evaluación similar a la inicial, valorando a los pacientes de los dos grupos que continuaban en el estudio para evaluar la eficacia real de la intervención llevada a cabo.

\section{Mediciones y criterios de evaluación:}

La información en las dos evaluaciones fue recogida por dos evaluadores que desconocían el grupo de asignación e independientes del equipo investigador.

El nivel de actividad física se estimó mediante el 7-PAR Day ${ }^{24}$, que proporciona una estimación del gasto energético operativizado en kilocalorías por kilogramo consumidas al día y la dosis de actividad física expresado en mets/hora/semana. Se considera activo si realiza actividad física de intensidad moderada al menos 30 minutos al día 5 días a la semana.

La calidad de vida relacionada con la salud física y mental se valoró mediante la versión española del SF-36 ${ }^{23}$. La alimentación se evaluó mediante una encuesta vali- dada para España de frecuencia alimentaria $^{29}$ para valorar el consumo de calorías, principios inmediatos, sal y adaptación a la dieta mediterránea. La fase de motivación en la que se encuentran para la realización de la dieta y el ejercicio se valoró siguiendo el modelo de Prochasca y Diclemente ${ }^{26}$.

Las mediciones de presión arterial se realizaron mediante el aparato OMROM M7®, siguiendo las recomendaciones de la Sociedad Europea de Hipertensión ${ }^{30}$. El peso se determinó con la balanza Seca 770 homologada, con el individuo descalzo y en ropa ligera y las lecturas se redondearon a 100 g. La circunferencia de cintura se midió con cinta métrica flexible en el punto medio entre la última costilla y la cresta ilíaca, con la cinta paralela al suelo, tomando la medida después de la inspiración. Las determinaciones de los niveles lipídicos y de glucosa en sangre fueron realizadas de manera ciega por el servicio de laboratorio asociado al centro de salud, tras al menos 12 horas de ayuno con extracciones en $2.006 \mathrm{y}$ 2.008 , sin congelar las muestras. Los datos demográficos, los factores de riesgo cardiovascular, las enfermedades cardiovasculares y los tratamientos antihipertensivos e hipolipemiantes fueron obtenidos de la historia clínica del paciente. Para la evaluación del riesgo cardiovascular se utilizó la escala de Framingham, versión Grundy de 1999, que estima el riesgo coronario absoluto a 10 años ${ }^{22}$.

Análisis estadístico: Las variables cuantitativas han sido expresadas con su media y desviación estándar (DS) y las cualitativas según su distribución de frecuencias. Se ha utilizado el test de $\chi^{2}$ de Pearson para analizar la asociación de variables cualitativas independientes y para valorar los cambios en el tiempo el test de McNemar. Se ha utilizado la prueba $\mathrm{T}$ de Student para muestras relacionadas o independientes, según el caso, para la comparación de medias de dos grupos. Para el contraste de hipótesis se fijó un riesgo $\alpha$ de 0,05. El análisis se realizó 
por intención de tratar. La eficacia de la intervención se ha evaluado comparando las diferencias que han experimentado ambos grupos antes y después de la intervención y se expresaron con un intervalo de confianza del $95 \%$ a través de la siguiente expresión:

[(media final-media basal en grupo de estudio)-( media final-media basal en grupo de control)].

El paquete estadístico utilizado fue el SPSS/PC+ (V. 15.0).

\section{RESULTADOS}

Las características basales de la población de estudio se muestran en la tabla 1 , en la que no existen diferencias estadísticamente significativas en ninguno de los parámetros evaluados entre ambos grupos. La edad media fue de 65 años, encontrando un consumo medio de sal superior a 3 gr./día, alta ingesta calórica y un buen control de la presión arterial, estando los valores medios por debajo de 140/90 $\mathrm{mmHg}$.

Tabla 1

Características basales de los pacientes según el grupo. Edad, sexo, factores de riesgo cardiovascular, dieta y ejercicio físico

\begin{tabular}{|c|c|c|c|c|}
\hline & & Intervención $\mathbf{n}=\mathbf{5 1}$ & Control $\mathbf{n}=\mathbf{5 0}$ & $\mathbf{p}$ \\
\hline \multirow{3}{*}{ Edad, años media $\pm \mathrm{DE}$} & Global & $64,5 \pm 9,7$ & $65,4 \pm 8,4$ & 0,425 \\
\hline & Varones & $60,9 \pm 12,2$ & $66,6 \pm 8,4$ & 0,120 \\
\hline & Mujeres & $67,4 \pm 6,0$ & $65,66 \pm 7,4$ & 0,317 \\
\hline \multicolumn{2}{|l|}{ Mujeres, $\mathrm{n}(\%)$} & $28(56 \%)$ & $34(68 \%)$ & 0,303 \\
\hline \multicolumn{2}{|l|}{ Diabetes mellitus, n (\%) } & $4(9,1 \%)$ & $3(6,8 \%)$ & 0,904 \\
\hline \multicolumn{2}{|l|}{ Obesidad (IMC $>30), \mathrm{n}(\%)$} & $18(36 \%)$ & $20(40 \%)$ & 0,837 \\
\hline \multicolumn{2}{|l|}{ Tabaco, n, $(\%)$} & $6(13 \%)$ & $1(2 \%)$ & 0,054 \\
\hline \multicolumn{2}{|c|}{ Antecedentes de enfermedades cerebrovascular n (\%) } & $1(2 \%)$ & $1(2 \%)$ & 0,748 \\
\hline \multicolumn{2}{|c|}{ Enfermedad arterial periférica $\mathrm{n}(\%)$} & $2(3,9 \%)$ & $1(2 \%)$ & 0,054 \\
\hline \multicolumn{2}{|l|}{ Fármacos para la PA, media \pm DE } & $1,4 \pm 0,8$ & $1,4 \pm 0,9$ & 0,906 \\
\hline \multicolumn{2}{|l|}{ Fármacos antihipertensivos n (\%) } & $43(84,3 \%)$ & $44(88 \%)$ & 0,403 \\
\hline \multicolumn{2}{|l|}{ Terapia combinada n, (\%) } & $26(51 \%)$ & $20(40 \%)$ & 0,198 \\
\hline \multicolumn{2}{|l|}{ Fármacos hipolipemiantes n, (\%) } & $9(17,8 \%)$ & $5(10 \%)$ & 0,206 \\
\hline \multirow{2}{*}{ Presión arterial, mmHg media $\pm \mathrm{DE}$} & Sistólica & $136,8 \pm 19,0$ & $139,3 \pm 17,7$ & 0,521 \\
\hline & Diastólica & $82,7 \pm 11,6$ & $79,3 \pm 10,5$ & 0,130 \\
\hline \multicolumn{2}{|l|}{$\mathrm{IMC}$, media $\pm \mathrm{DE}$} & $29,3 \pm 4,7$ & $29,4 \pm 4,9$ & 0,859 \\
\hline \multicolumn{2}{|c|}{ Circunferencia de la cintura, media $\pm \mathrm{DE}$} & $100,1 \pm 13,1$ & $100,1 \pm 11,3$ & 0,981 \\
\hline \multicolumn{2}{|c|}{ Dosis de actividad física, media $\pm \mathrm{DE}$} & $9,4 \pm 16,9$ & $10,7 \pm 16,8$ & 0,691 \\
\hline \multicolumn{2}{|l|}{ Riesgo coronario absoluto, media $\pm \mathrm{DE}$} & $11,1 \pm 6,6$ & $12,3 \pm 7,4$ & 0,373 \\
\hline \multicolumn{2}{|l|}{ Hidratos de carbono gr/día, media \pm DE } & $273,6 \pm 86,3$ & $313,0 \pm 127,1$ & 0,072 \\
\hline \multicolumn{2}{|l|}{ Grasas gr/día, media $\pm \mathrm{DE}$} & $109,4 \pm 52,3$ & $112,9 \pm 45,5$ & 0,444 \\
\hline \multicolumn{2}{|l|}{ Proteínas gr/día, media \pm DE } & $119,84 \pm 56,01$ & $117,9 \pm 38,5$ & 0,843 \\
\hline \multicolumn{2}{|l|}{ Cloruro Na gr/día, media \pm DE } & $3,4 \pm 1,0$ & $3,6 \pm 1,6$ & 0,340 \\
\hline \multicolumn{2}{|l|}{ Alcohol gr/día, media \pm DE } & $10,8 \pm 15,2$ & $9,7 \pm 17,5$ & 0,742 \\
\hline \multicolumn{2}{|c|}{ Consumo de Kilocarias/día, media \pm DE } & $2633 \pm 705$ & $2.838 \pm 1038$ & 0,250 \\
\hline \multicolumn{2}{|l|}{ Pacientes activos, $\mathrm{n}(\%)$} & $16(26 \%)$ & $11(22 \%)$ & 0,408 \\
\hline \multirow{2}{*}{ SF 36, media \pm DE } & Salud Física & $51,0 \pm 7,0$ & $50,4 \pm 6,4$ & 0,696 \\
\hline & Salud Mental & $51,0 \pm 12,1$ & $53,1 \pm 9,6$ & 0,337 \\
\hline \multirow{4}{*}{ Colesterol, mg/dl media $\pm \mathrm{DE}$} & Total & $213,3 \pm 41,3$ & $218,7 \pm 33,6$ & 0,473 \\
\hline & LDL & $127,1 \pm 35,4$ & $136,2 \pm 27,1$ & 0,160 \\
\hline & HDL & $60,0 \pm 14,2$ & $62,7 \pm 16,0$ & 0,532 \\
\hline & Triglicéridos & $100,1 \pm 13,1$ & $107,1 \pm 48,7$ & 0,060 \\
\hline
\end{tabular}

IMC: Índice de masa corporal. SF 36: Cuestionario de calidad de vida estandarizado para población española. 
La tabla 2 muestra las diferencias entre la evaluación final y basal en cada uno de los grupos, así como, la eficacia de la intervención en los factores de riesgo, riesgo cardiovascular y fármacos. Únicamente se encuentra diferencias entre la evaluación final y basal en la presión arterial diastólica en el grupo de intervención $(-3,9 \mathrm{mmHg}$;

Tabla 2

Efectos de la intervención en los factores de riesgo cardiovascular, en el riesgo coronario absoluto y en el tratamiento

\begin{tabular}{|c|c|c|c|c|c|c|c|c|}
\hline & \multicolumn{3}{|c|}{ Grupo Intervención (GI) } & \multicolumn{3}{|c|}{ Grupo Control (GC) } & \multirow{2}{*}{\begin{tabular}{|c|} 
Efectividad \\
$\begin{array}{c}\text { Grupo Estudio-Grupo Control } \\
\text { (IC 95\%) }\end{array}$
\end{tabular}} \\
\hline & & Basal & Final & $\begin{array}{l}\text { Diferencias } \\
\text { media } \pm \text { DE }\end{array}$ & Basal & Final & $\begin{array}{l}\text { Diferencias } \\
\text { media } \pm \text { DE }\end{array}$ & \\
\hline \multicolumn{2}{|c|}{ PAS mmHg } & 137,9 & 132,6 & $-5,3 \pm 19,6$ & 139,1 & 132,0 & $-7,1 \pm 16,3^{*}$ & $1,9(-5,5 \div 9,3)$ \\
\hline \multicolumn{2}{|c|}{ PAD mmHg } & 83,8 & 79,9 & $-3,9 \pm 10,8 *$ & 79,6 & 76,9 & $-2,7 \pm 11,5$ & $-1,2(-5,6 \div 9,3)$ \\
\hline \multirow{4}{*}{ Colesterol } & Total & 213,7 & 211,0 & $-2,7 \pm 36,2$ & 217,8 & 216,7 & $-1,1 \pm 29,0$ & $-1,6(-15,1 \div 11,8)$ \\
\hline & LDL & 128,0 & 128,7 & $0,7 \pm 32,2$ & 134,9 & 134,4 & $-0,5 \pm 26,8$ & $1,2(-11,2 \div 13,6)$ \\
\hline & HDL & 60,6 & 58,0 & $-2,5 \pm 11,9$ & 62,7 & 60,6 & $-2,2 \pm 8,9$ & $-0,35(-4,7 \div 4,0)$ \\
\hline & Triglicéridos & 130,8 & 126,9 & $-4,0 \pm 7,2$ & 108,5 & 115,2 & $6,7 \pm 32,3$ & $-10,7(-27,6 \div 6,2)$ \\
\hline \multicolumn{2}{|l|}{ IMC } & 29,5 & 29,2 & $-0,3 \pm 1,6$ & 29,7 & 29,8 & $0,1 \pm 1,5$ & $-0,4(-1,1 \div 0,3)$ \\
\hline \multicolumn{2}{|c|}{ Circunferencia de la cintura } & 101,3 & 100,4 & $-0,9 \pm 6,8$ & 100,7 & 101,3 & $0,6 \pm 5,3$ & $-1,5(-4,1 \div 1,0)$ \\
\hline \multicolumn{2}{|c|}{ Riesgo coronario Absoluto } & 11,1 & 10,4 & $-0,8 \pm 6,5$ & 12,45 & 12,7 & $0,2 \pm 6,8$ & $-1,0(-3,9 \div 1,9)$ \\
\hline \multicolumn{2}{|c|}{ Fármacos para la PA media } & 1,38 & 1,47 & $0,09 \pm 0,66$ & 1,38 & 1,56 & $0,17 \pm 0,5$ & $-0,08(-0,32 \div 0,16)$ \\
\hline \multicolumn{2}{|c|}{ Fármacos Hiperlipemia } & 0,18 & 0,24 & $0,06 \pm 0,33$ & 0,08 & 0,17 & $0,08 \pm 0,28$ & $-0,02(-0,14 \div 0,11)$ \\
\hline
\end{tabular}

PAS: Presión arterial sistólica. PAD: Presión arterial diastólica. IMC: Índice de masa corporal. Efectividad: [(media final-media basal en grupo de estudio)( media final-media basal en grupo de control)]. IMC: Índice de masa corporal. * p-valor $<0,05$

Tabla 3

Efectos de la intervención sobre el consumo de principios inmediatos, sal, alcohol y kilocalorías totales

\begin{tabular}{|c|c|c|c|c|c|c|c|}
\hline & & upo Interv & ención (GI) & & rupo Con & trol (GC) & Efectividad \\
\hline & Basal & Final & $\begin{array}{l}\text { Diferencias } \\
\text { media } \pm \text { DE }\end{array}$ & Basal & Final & $\begin{array}{l}\text { Diferencias } \\
\text { media } \pm \text { DE }\end{array}$ & $\begin{array}{l}\text { Efecto de la intervención } \\
\text { (IC 95\%) }\end{array}$ \\
\hline Energía (Kcal/día) & $2.737,42$ & $2.652,76$ & $-84,68 \pm 1141,78$ & 2730,45 & $2.483,04$ & $-247,40 \pm 1120,91$ & $162,7(-306 \div 631)$ \\
\hline H. carbono (gr/dia) & 293,50 & 288,65 & $-4,84 \pm 111,75$ & 297,98 & 275,82 & $-22,16 \pm 129,86$ & $17,31(-32,8 \div 67,49)$ \\
\hline Grasas (gr/dia) & 113,13 & 105,01 & $-8,16 \pm 60,94$ & 111,12 & 100,38 & $-10,73 \pm 57,49$ & $2,6(-21,92 \div 27,16)$ \\
\hline Proteínas (gr/dia) & 116,98 & 114,86 & $-2,12 \pm 82,89$ & 118,40 & 103,50 & $-14,90 \pm 46,40^{*}$ & $12,78(-15 \div 40,6)$ \\
\hline Fibra (gr/dia) & 28,78 & 28,52 & $-0,25 \pm 13,62$ & 33,51 & 29,03 & $-4,47 \pm 14,67 *$ & $4,2(-1,6 \div 10)$ \\
\hline Colesterol (mg/dia) & 491,27 & 494,25 & $3,01 \pm 487,93$ & 496,68 & 420,46 & $-76,22 \pm 352,34$ & $79,23(-97 \div 255)$ \\
\hline AGM (gr/dia) & 50,38 & 47,93 & $-2,44 \pm 26,89$ & 49,67 & 44,78 & $-4,86 \pm 24,15$ & $2,4(-8,1 \div 13)$ \\
\hline AGP (gr/dia) & 19,64 & 18,95 & $-0,68 \pm 16,96$ & 17,98 & 17,79 & $-0,19 \pm 9,60$ & $-0,49(-6,2 \div 5,21)$ \\
\hline AGS (gr/dia) & 32,32 & 28,38 & $-3,94 \pm 17,08$ & 33,94 & 28,28 & $-5,66 \pm 28,12$ & $1,7(-7,9 \div 11,35)$ \\
\hline Cloruro $\mathrm{Na}(\mathrm{mg} /$ dia $)$ & 3430 & 3449 & $18,60 \pm 1654$ & 3639 & 3348 & $-290,2 \pm 1857$ & $308(-419 \div 1037)$ \\
\hline Alcohol (g/dia) & 11,04 & 13,36 & $2,32 \pm 18,45$ & 9,26 & 8,89 & $-0,36 \pm 11,03$ & $2,7(-3,6 \div 8,9)$ \\
\hline Verduras, hortalizas y frutas (gr/dia) & 896,77 & 858,48 & $-38,28 \pm 647,85$ & 1013,56 & 825,65 & $-187,90 \pm 519,03 *$ & $149(-93,5 \div 392,7)$ \\
\hline Legumbres y derivados (gr/dia) & 25,27 & 19,46 & $-5,80 \pm 16,52 *$ & 27,52 & 21,35 & $-6,17 \pm 28,07$ & $0,36(-9,17 \div 9,90)$ \\
\hline Carne (gr/dia) & 186,12 & 157,58 & $-28,53 \pm 199,61$ & 182,57 & 147,65 & $-34,92 \pm 130,17$ & $6,4(-63,4 \div 76,2)$ \\
\hline Pescado (gr/dia) & 127,02 & 166,02 & $39,00 \pm 276,12$ & 109,54 & 106,74 & $-2,79 \pm 56,69$ & $41,80(-40,7 \div 124,3)$ \\
\hline Lácteos y derivados (gr/dia) & 462,47 & 503,53 & $41,06 \pm 244,46$ & 561,30 & 497,88 & $-63,42 \pm 272,20$ & $104,5(-2,68 \div 211)$ \\
\hline Aceite oliva (gr/dia) & 30,43 & 31,84 & $1,41 \pm 20,56$ & 28,97 & 27,09 & $-1,88 \pm 18,23$ & $3,3(-4,7 \div 11,3)$ \\
\hline Frutos secos (gr/dia) & 15,02 & 10,00 & $-5,02 \pm 31,10$ & 9,23 & 11,70 & $2,47 \pm 12,82$ & $-7,49(-17,62 \div 2,63)$ \\
\hline Adaptación dieta mediterránea & $59,2 \%$ & $59,6 \%$ & $0,3 \pm 12,22$ & $59,9 \%$ & $56,4 \%$ & $-3,5 \pm 15,6$ & $3,8(-2,1 \div 9,7)$ \\
\hline
\end{tabular}

Consumo dieta: expresado en gr/día. Efectividad: [(media final-media basal en grupo de estudio)-(media final-media basal en grupo de control)] AGM: Ácidos grasos momoinsaturados, AGP: Ácidos grasos piliinsaturados, AGS: Ácidos grasos saturados. * p-valor $<0,05$ 
IC95\%:-7,1 - -0,65) y la sistólica en el grupo control (-7,1 mmHg; IC95\%: -11,8 $-2,42)$. No se encuentran diferencias en la eficacia de la intervención al comparar los cambios entre el grupo de intervención y el grupo de control. No obstante, se ha encontrado un descenso del riesgo cardiovascular en el grupo de intervención de 0,8 puntos porcentuales frente a un ascenso de 0,2 en el grupo control, lo que supone un efecto de 1 punto (IC95\%: -3,9 - 1,9) de descenso en el riesgo, sin que alcance significación estadística.

En relación a la ingesta calórica, consumo de principios inmediatos, sal y alcohol, no se encuentran diferencias estadística- mente significativas entre la evaluación basal y final, ni en la eficacia de la intervención, excepto un descenso en el consumo de proteínas (14,9 g/día), fibra (4,4 gr/día) y verduras/frutas $(187,9 \mathrm{gr} /$ día $)$ en el grupo control y de legumbres o derivados $(-5,8$ gr/día) en el grupo de intervención. La adaptación a la dieta mediterránea es favorable al grupo de intervención con un incremento de 3,8 (IC95\%: -2,1 - 9,7) puntos porcentuales, respecto al control (tabla 3 ). Tampoco se han encontrado diferencias entre los grupos en la calidad de vida, ni en el área física ni en la mental. Se ha observado un incremento tanto en la dosis de actividad física como el porcentaje de pacientes activos en los dos grupos, sin alcanzar la

Tabla 4

Efectos de la intervención sobre el ejercicio físico y la calidad de vida

\begin{tabular}{|c|c|c|c|c|c|c|c|c|}
\hline & & \multicolumn{3}{|c|}{ Grupo Intervención (GI) } & \multicolumn{3}{|c|}{ Grupo Control (GC) } & \multirow{2}{*}{\begin{tabular}{|c|} 
Efectividad \\
$\begin{array}{c}\text { Grupo Estudio-Grupo Control } \\
\text { (IC 95\%) }\end{array}$ \\
\end{tabular}} \\
\hline & & Basal & Final & $\begin{array}{r}\text { Diferencias } \\
\text { media } \pm \text { DE }\end{array}$ & Basal & Final & $\begin{array}{l}\text { Diferencias } \\
\text { media } \pm \text { DE }\end{array}$ & \\
\hline \multicolumn{2}{|c|}{$\begin{array}{l}\text { Dosis actividad física } \\
\text { (mets/hora/semana) }\end{array}$} & 9,8 & 13,5 & $3,6 \pm 19,0$ & 10,1 & 14,0 & $3,9 \pm 14,9$ & $-0,3(-7,3-6,7)$ \\
\hline \multicolumn{2}{|c|}{ Gasto (Klc/kilo/dia) } & 32,9 & 33,6 & $0,7 \pm 2,1^{*}$ & 32,9 & 33,5 & $0,6 \pm 1,4^{*}$ & $-30,7(-104,8-43,39)$ \\
\hline \multicolumn{2}{|c|}{ Pacientes activos \% } & 26,7 & 35,6 & 8,8 & 20,8 & 35,4 & 14,5 & $-5,6(-29,9-18,6)$ \\
\hline \multirow{2}{*}{ SF 36} & Salud Física & 51,3 & 48,5 & $-2,8 \pm 8,9$ & 50,4 & 48,5 & $-1,9 \pm 7,3$ & $-1,0(-4,3-2,4)$ \\
\hline & Salud Mental & 51,0 & 52,6 & $1,6 \pm 11,3$ & 53,0 & 53,0 & $-0,0 \pm 11,6$ & $1,6(-3,2-6,3)$ \\
\hline
\end{tabular}

Efectividad: [(media final-media basal en grupo de estudio)-( media final-media basal en grupo de control)]. SF 36: Cuestionario de calidad de vida estandarizado para población española. * p-valor $<0,05$.

Tabla 5

Variación en la fase de motivación del paciente para realizar ejercicio y cambiar los hábitos de su dieta

\begin{tabular}{|c|c|c|c|c|c|c|c|c|}
\hline & & \multicolumn{3}{|c|}{ Grupo Intervención (GI) } & \multicolumn{3}{|c|}{ Grupo Control (GC) } & \multirow{2}{*}{\begin{tabular}{|c|} 
Efectividad \\
$\begin{array}{c}\text { Grupo Estudio-Grupo Control } \\
\text { (IC 95\%) }\end{array}$ \\
\end{tabular}} \\
\hline & & Basal & Final & $\begin{array}{l}\text { Diferencias } \\
\text { media } \pm \mathrm{DE}\end{array}$ & Basal & Final & $\begin{array}{l}\text { Diferencias } \\
\text { media } \pm \text { DE }\end{array}$ & \\
\hline \multicolumn{9}{|c|}{ Motivación } \\
\hline \multirow[t]{5}{*}{ Ejercicio } & Precontemplación & 30,7 & 35,9 & 5,1 & 25,6 & 30,2 & 4,6 & $0,5(-14,9-15,9)$ \\
\hline & Contemplación & 5,1 & 2,6 & $-2,5$ & 2,3 & 4,6 & 2,3 & $-4,8(-11,7-1,9)$ \\
\hline & Preparación & 2,6 & 2,6 & 0,0 & 2,3 & 2,3 & 0,0 & $0(-6,9-6,9)$ \\
\hline & Acción & 10,3 & 5,1 & $-5,2$ & 2,3 & 4,6 & 2,3 & $-7,5(-22,4-7,5)$ \\
\hline & Mantenimiento & 51,3 & 53,8 & 2,5 & 67,4 & 58,1 & $-9,3$ & $11,8(-0,8-31,9)$ \\
\hline \multicolumn{9}{|c|}{ Motivación } \\
\hline \multirow[t]{5}{*}{ Dieta } & Precontemplación & 24,4 & 19,5 & $-4,9$ & 21,4 & 26,2 & 4,7 & $-9,6(-32,3-13,1)$ \\
\hline & Contemplación & 2,4 & 0,0 & $-2,4$ & 4,7 & 9,5 & 4,7 & $-7,2(-19,9-5,5)$ \\
\hline & Preparación & 0,0 & 2,4 & 2,4 & 0,0 & 0,0 & 0,0 & $2,4(-2,4-7,3)$ \\
\hline & Acción & 4,8 & 2,4 & $-2,4$ & 0,0 & 2,4 & 2,4 & $-4,8(-14,5-4,9)$ \\
\hline & Mantenimiento & 68,3 & 75,6 & 7,3 & 73,8 & 61,2 & $-11,9$ & $19,2(-4,1-42,5)$ \\
\hline
\end{tabular}

Efectividad: [(media final-media basal en grupo de estudio)-(media final-media basal en grupo de control)] 
Figura 1

Esquema general del estudio

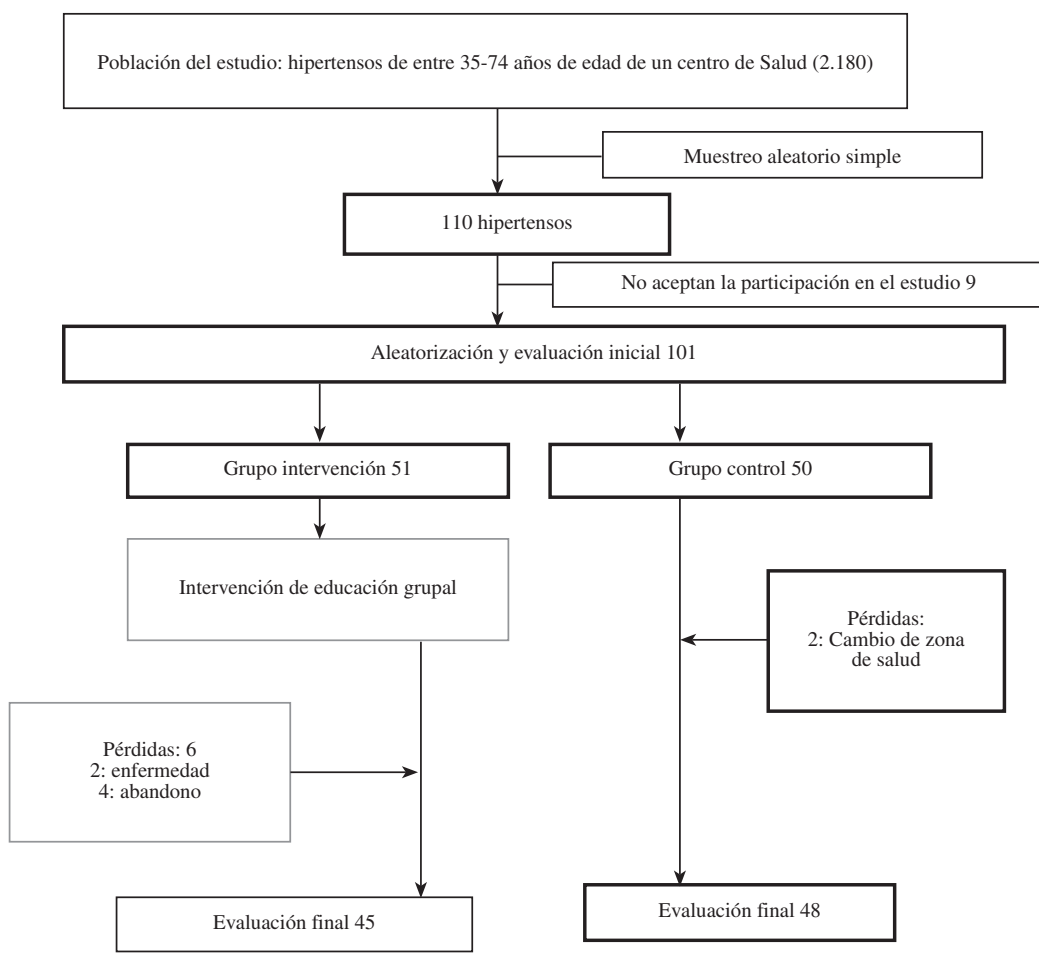

significación estadística. Sin embargo se han encontrado diferencias significativas en los dos grupos en el gasto calórico/kilo al día que realizan los pacientes entre la evaluación basal y final (tabla 4).

Respecto a las fases de motivación, ha disminuido el porcentaje de pacientes que se encuentran en fase de precontemplación en la dieta cardiosaludable y aumentando los que están en fase de mantenimiento tanto en dieta como ejercicio en el grupo de intervención, mientras que, en el grupo control sucede lo contrario (Tabla 5).

\section{DISCUSIÓN}

En la evaluación del efecto de la intervención, aunque no se han encontrado dife-

rencias estadísticamente significativas entre ambos grupos, se ha observado una tendencia a mejorar más el grupo de intervención que el control. Este efecto se puede observar con claridad en el riesgo cardiovascular, como variable globalizadota de la evaluación, en el que se observa una disminución de 0,8 puntos en el grupo de intervención, mientras que el grupo control aumenta 0,2 puntos.

Esa misma tendencia, se ha encontrado también, en el cambio de la fase de motivación para modificar la dieta y el ejercicio, en la mejor adaptación a la dieta mediterránea, y en el menor incremento del número de fármacos utilizados en el tratamiento de la hipertensión arterial. No obstante, se ha encontrado una tendencia de mejora similar en los dos grupos en el incremento de ejer- 
cicio físico y la disminución de la ingesta calórica. La obtención de pocos resultados efectivos con la intervención educativa, en las variables objeto de la evaluación, probablemente ha sido debido la dificultad que conlleva modificar hábitos de conducta en las personas adultas muy influenciados por relaciones sociales, sin olvidar que la mejoría en muchas de las variables analizadas en los dos grupos disminuye la diferencia del efecto de la intervención.

Como limitaciones del estudio, hay que tener en cuenta en primer lugar, el tamaño de la muestra (50 pacientes por grupo) que disminuye la potencia del estudio, y probablemente es la causa de que a pesar de haber seguido un proceso minucioso en la aleatorización, existieran diferencias, aunque no estadísticamente significativas, en algunas variables en la evaluación basal. El hecho de que los pacientes pertenezcan a un solo centro de salud limita la validez externa. Por otra parte, no podemos olvidar la posible contaminación, ya que, son pacientes que viven en la misma zona, y el simple hecho de la inclusión en el estudio y la evaluación basal realizada puede haber tenido su efecto en las modificaciones de estilos de vida en el grupo control. Por último, el intentar valorar todos los factores que han demostrado eficacia para disminuir la presión arterial, hace que tanto el diseño y sobre todo la intervención sean demasiado complejas pudiendo diluirse la eficacia de la misma.

Los resultados obtenidos en este estudio en relación a la presión arterial son similares a los encontrados por otros autores en diferentes intervenciones educativas sobre pacientes hipertensos ${ }^{16,31,32}$. Por lo que, parece necesario acompañar a las estrategias educativas de otro tipo de intervenciones complementarias para lograr un descenso efectivo de la presión arterial.

Las intervenciones encaminadas a aumentar el ejercicio físico, según muestran varios ensayos clínicos han llegado a conclusiones diferentes ${ }^{33-35}$. Una revisión de las intervenciones diseñadas para aumentar la actividad física concluye que pueden ser moderadamente efectivas al incentivar a las personas a ser físicamente activas y a tener un mejor estado físico. El asesoramiento profesional y la orientación, junto con el apoyo constante, pueden favorecer el incremento de la actividad física ${ }^{36}$. Sin embargo, la mayoría de los estudios no duraron más de un año, y tanto la forma de medición del ejercicio, las intervenciones realizadas y las poblaciones a las que iban dirigidas eran muy heterogéneas, por lo que resulta difícil comparar los resultados entre ellos y con los nuestros.

No hemos encontrado trabajos que evalúen los cambios en la dieta en pacientes hipertensos tras una intervención educativa. No obstante, las intervenciones que se han mostrado efectivas para afrontar un cambio en la dieta, son aquellas que combinan la educación nutricional con el consejo conductual orientado a ayudar a adquirir habilidades, motivación y apoyo necesario para cambiar su patrón dietético diario ${ }^{37}$.

El menor incremento del número de fármacos utilizados en el tratamiento de la hipertensión en el grupo de intervención en la evaluación final, puede sugerir que la intervención ha sido más efectiva que lo que reflejan los niveles de presión arterial, en el mismo sentido va el cambio de la fase de motivación en el ejercicio y en los hábitos dietéticos.

Por todo ello, la utilización exclusiva de intervenciones educativas dirigidas a pacientes hipertensos tiene pocas probabilidades de conseguir reducciones clínicamente importantes de la presión arterial. En este tipo de intervención es importante valorar el riesgo cardiovascular y la fase de motivación en la cual se encuentre el paciente ya que el abordaje debe ser diferente. 
Por ultimo, las actividades de educación grupal consumen muchos recursos y los resultados son inciertos. Por lo que sería recomendable continuar investigando en el desarrollo de estrategias más efectivas, probablemente más duraderas con seguimiento y evaluación continuada antes de generalizar este tipo de actividades en pacientes hipertensos sin haber demostrado su eficacia.

\section{AGRADECIMIENTOS}

Queremos agradecer la colaboración de los médicos/as y enfermeras/os del equipo de atención primaria participante y a las personas hipertensas por su colaboración.

\section{BIBLIOGRAFÍA}

1. Banegas Banegas JR, Rodriguez-Artalejo F, De La Cruz Troca JJ, De Andres Manzano B, Del Rey Calero J. Mortalidad relacionada con la hipertensión y la presión arterial en España. Med Clin (Barc). 1999; 112: 489-94.

2. Llisterri Caro JL, Rodriguez Roca GC, Alonso Moreno FJ, Banegas Banegas JR, Gonzalez-Segura Alsina D, Lou Arnal S, et al. Control de la presión arterial en la población hipertensa española atendida en Atención Primaria. Estudio PRESCAP 2006 Study. Med Clin (Barc). 2008; 130: 681-7.

3. Llisterri Caro JL, Rodriguez Roca GC, Alonso Moreno FJ, Lou Arnal S, Divison Garrote JA, Santos Rodriguez JA, et al. Control de la presión arterial en la población hipertensa española atendida en Atención Primaria. Estudio PRESCAP 2002. Med Clin (Barc). 2004; 122: 165-71.

4. Whelton PK, He J, Appel LJ, Cutler JA, Havas S, Kotchen TA, et al. Primary prevention of hypertension: Clinical and public health advisory from The National High Blood Pressure Education Program. JAMA. 2002; 288: 1882-1888.

5. Steven VJ, Obarzanek E, Cook NR, Lee IM, Appel LJ, Smith West et al. Long-term weight loss and changes in blood pressure: results of the trials of hypertension prevention, phase II. Ann Intern Med. 2001; 134: 1-11.
6. Whelton SP, Chin A, Xin X, He J. Effect of Aerobic Exercise on Blood Pressure: A Meta-Analysis of Randomized, Controlled Trials. Ann Intern Med. 2002; 136: 493-503.

7. Xin X, He J, Frontini MG, Ogden LG, Motsamai OI, Whelton PK., et al. Effects of alcohol reduction on blood pressure: a meta-analysis of randomized controlled trials. Hypertension, 2001; 38: 1112-1117.

8. Singh RB, Rastogi SS, Verma R, Laxmi B, Singh $\mathrm{R}$, Ghosh S, et al., Randomized controlled trial of cardioprotective diet in patients with recent acute myocardial infarction: results of one year follow up. BMJ, 1992; 304: 1015-1019.

9. He FJ, MacGregor GA. Efecto de la reducción moderada de sal a largo plazo en la presión arterial (Revisión Cochrane traducida). En: La Biblioteca Cochrane Plus, 2005 Número 4. Oxford: Update Software Ltd. Disponible en: http://www.updatesoftware.com. (Traducida de The Cochrane Library, 2005 Issue 4. Chichester, UK: John Wiley \& Sons, Ltd.).

10. Chobanian AV, Bakris GL, Black HR, Cushman WC, Green LA, Izzo JL, et al. The Seventh Report of the Joint National Committee on Prevention, Detection, Evaluation, and Treatment of High Blood Pressure: The JNC 7 Report. JAMA. 2003; 289: 2560-72.

11. Gómez Marcos MA, Garcia Ortiz L, Gonzalez Elena LJ, Ramos Delgado E, Gonzalez Garcia A, Parra Sanchez J. Efectividad de una intervención de mejora de calidad en el control de la presión arterial en atención primaria. Rev Clin Esp. 2006; 206: 428-34.

12. Gómez Marcos MA, Garcia Ortiz L, Gonzalez Elena LJ, Sanchez Rodriguez A. Efectividad de una intervención de mejora de calidad en la reducción del riesgo coronario y del riesgo de mortalidad cardiovascular en pacientes hipertensos.. Aten Primaria. 2006; 37: 498-503.

13. García Ortiz L, Santos Rodriguez I, Gomez Marcos MA, Sanchez Fernandez PL, Rodriguez Sanchez E, Gonzalez Elena LJ. Los ciclos de mejora de calidad en la atención al paciente hipertenso. (CICLO-RISK STUDY). Rev Esp Salud Publica. 2008; 82: 57-68.

14. Garcia Ortiz L, Gomez Marcos MA, Gonzalez Elena LJ, Maderuelo Fernandez JA, Ramos Delgado E, Torrecilla Garcia M. Riesgo cardiovascular del paciente hipertenso con seguimiento prolongado en Atención Primaria. El efecto del envejecimiento (CICLO-RISK study) Rev Esp Salud Publica. 2007; 81: 365-73. 
15. Garcia Ortiz L, Santos Rodriguez I, Sanchez Fernandez PL, Mora Santiago MC, Arganda Maya J, Rodriguez Corral MT. Efectividad de una intervención de mejora de calidad en la reducción del riesgo cardiovascular en pacientes hipertensos. Rev Esp Cardiol. 2004; 57:644-51.

16. Fahey T, Schroeder K, Ebrahim S. Interventions Used to Improve Control of Blood Pressure in Patients with Hypertension. Cochrane Database Syst Rev. 2006(4): CD005182.

17. Executive Summary of the Third Report of the National Cholesterol Education Program (NCEP) Expert Panel on Detection, Evaluation, and Treatment of High Blood Cholesterol in Adults (Adult Treatment Panel Iii). JAMA. 2001 May; 285: 2486-97.

18. European Society of Hypertension-European Society of Cardiology Guidelines Committee. 2003 European Society of Hypertension-European Society of Cardiology Guidelines for the Management of Arterial Hypertension. J Hypertens. 2003; 21: 1011-53.

19. De Backer G, Ambrosioni E, Borch Johnsen K, Brotons C, Cifkova R, Dallongeville J, et al. European Guidelines on Cardiovascular Disease Prevention in Clinical Practice: Third Joint Task Force of European and Other Societies on Cardiovascular Disease Prevention in Clinical Practice (Constituted by Representatives of Eight Societies and by Invited Experts). Eur J Cardiovasc Prev Rehabil. 2003; 10: S1-S10.

20. Standards of Medical Care in Diabetes. Diabetes Care. 2004 Jan;27 Suppl 1:S15-35.

21. Salas Salvado J, Rubio MA, Barbany M, Moreno B. SEEDO 2007 Consenso para la evaluación del sobrepeso y la obesidad y criterios para el establecimiento de una intervención terapéutica. Med Clin (Barc). 2007 Feb 10; 128(5): 184-96;

22. Grundy SM, Pasternak R, Greenland P, Smith S, Fuster V. Assessment of Cardiovascular Risk by Use of Multiple-Risk-Factor Assessment Equations: A Statement for Healthcare Professionals from the American Heart Association and the American College of Cardiology. Circulation. 1999; 100: 1481-92.

23. Alonso J, Prieto L, Anto JM. [La versión en español de la SF-36 Health Survey (SF-36 Cuestionario de Salud): un instrumento para la medición de resultados clínicos]. Med Clin (Barc). 1995; 104: 771-6.

24. Sallis JF, Haskell WL, Wood PD, Fortmann SP, Rogers T, Blair SN, et al. Physical Activity
Assessment Methodology in the Five-City Project. Am J Epidemiol. 1985; 121:91-106.

25. Mochari H, Gao Q, Mosca L. Validation of the Medficts Dietary Assessment Questionnaire in a Diverse Population. J Am Diet Assoc. 2008; 108: 817-22.

26. Prochaska J, Dc. Transtheoretical Therapy: Towards a More Integrative Model of Change. Psychother Theor Res Pract Train. 1982; 19: 276.

27. Kris Etherton P, Eckel RH, Howard BV, St Jeor S, Bazzarre TL. Aha Science Advisory: Lyon Diet Heart Study. Benefits of a Mediterranean-Style, National Cholesterol Education Program/American Heart Association Step I Dietary Pattern on Cardiovascular Disease. Circulation. 2001; 103: 1823-5.

28. Estruch R, Martinez Gonzalez MA, Corella D, Salas Salvado J, Ruiz-Gutierrez V, Covas MI, et al. Effects of a Mediterranean-Style Diet on Cardiovascular Risk Factors: A Randomized Trial. Ann Intern Med. 2006; 145: 1-11.

29. Martín-Moreno JM, Boyle P, Gorgojo L, Maisonneuve P, Fernández-Rodríguez JC, Salvini S, et al. Development and validation of a food frequency questionnaire in Spain. Int J Epidemiol. 1993; 22: 512-9.

30. O'Brien E, Asmar R, Beilin L, Imai Y, Mancia G, Mengden $T$ et al. Practice guidelines of the european society of hypertension for clinic, ambulatory and self blood pressure measurement. J Hypertens. 2005; 23: 697-701.

31. Billault B, Degoulet P, Devries C, Plouin PF, Chatellier G, Menard J. Use of a Standardized Personal Medical Record by Patients with Hypertension: A Randomized Controlled Prospective Trial. MD Comput. 1995; 12: 31-5.

32. Martinez Amenos A, Fernandez Ferre ML, Mota Vidal C, Alsina Rocasalbas J. Evaluation of Two Educative Models in a Primary Care Hypertension Programme. J Hum Hypertens. 1990; 4: 362-4.

33. Effects of Physical Activity Counseling in Primary Care: The Activity Counseling Trial: A Randomized Controlled Trial. Writing Group for the Activity Counseling Trial Research Group. JAMA. 2001; 286: 677-87.

34. Van Sluijs EM, Van Poppel MN, Twisk JW, Chin Apmj, Calfas KJ, Van Mechelen W. Effect of a Tailored Physical Activity Intervention Delivered in General Practice Settings: Results of a Randomized Controlled Trial. Am J Public Health. 2005; 95: 1825-31. 
35. Elley CR, Kerse N, Arroll B, Robinson E. Effectiveness of Counselling Patients on Physical Activity in General Practice: Cluster Randomised Controlled Trial. BMJ. 2003; 326: 793.

36. Hillsdon M, Foster C, Thorogood M. Interventions for Promoting Physical Activity. Cochrane Database Syst Rev. 2005(1):CD003180.
37. Calfas KJ, Zabinski MF, Rupp J. Practical Nutrition Assessment in Primary Care Settings: A Review. Am J Prev Med. 2000; 18: 289-99. 